Originales

\title{
Valor pronóstico de los marcadores tumorales de proliferación celular y angiogénesis en los oligodendrogliomas
}

\author{
A. López-Muñiz ${ }^{*}$ J. C. Gutiérrez ${ }^{* *}$, C. García-Fernández ${ }^{* *}$, A. Herrero***
}

\section{Resumen}

- Propósito: la evolución clínica de los oligodendrogliomas (ODG) es impredecible por los actuales criterios clínicos e histológicos. La presencia de marcadores tumorales del ciclo celular o de la angiogénesis podría predecir el curso clínico de los ODG. El propósito de este estudio ha sido la cuantificación de las expresiones ki67 y CD34 en ODG y su correlación con el grado histológico y la supervivencia.

- Material y métodos: hemos estudiado 25 ODG. Las inmunoexpresiones ki67 y CD34 fueron determinadas por técnicas inmunohistoquímicas. Posteriormente se realizó un análisis cuantitativo con un programa de análisis de imagen sistema Qwin. Finalmente, se realizaron las cuantificaciones del ki67 y del CD34, y el análisis estadístico de los resultados.

- Resultados: el número de núcleos Ki67 positivos se correlacionó con la malignidad de la lesión. El número de núcleos ki67 también se correlacionó con la supervivencia de los pacientes. Por el contrario, el número de microvasos (estructuras CD34 positivas) no se correlacionaba ni con el grado histológico ni con la supervivencia en los ODG.

- Conclusiones: estos resultados sugieren que el ki67, fundamentalmente el número de núcleos marcados, es un importante indicador diagnóstico y puede ser usado como un parámetro de mal pronóstico en los ODG. Por el contrario, la angiogénesis no es un parámetro pronóstico en los ODG.

Palabras clave:

Oligodendroglioma. Angiogénesis. Proliferación celular. Antígeno Ki67. Antígeno CD34.

Oncología, 2004; 27 (5):289-296

\footnotetext{
* Departamento de Morfología y Biología Celular. Facultad de Medicina.

** Servicios de Neurología y Neurocirugía. Hospital Universitario Central de Asturias.

*** Departamento de Cirugía (Anatomía Patológica). Facultad de Medicina. HUCA.
}

Universidad de Oviedo. 


\section{Summary}

- Purpose: The clinical behavior of oligodendroglioma (ODG) is unpredictable using clinical and histological criteria. The present tumor markers related to cell cycle regulation and angiogenesis might improve the possibility of predicting the clinical course of ODG. The aim of this study is the quantification of ki67 and CD34 expression in oligodendrogliomas and its correlation with histological grade and survival.

- Material and methods: We studied 25 ODG. The ki67 and CD34 immunoexpression was determined by immunohistochemical techniques. Later, the quantitative study was carried out with a Qwin system image analysis program. Finally, ki67 and CD34 expression was quantified and a statistical analysis of the results was performed.

- Results: The number of ki67 positive nuclei correlated with the malignancy of the lesion, and with the overall survival. Contrarily, the number of CD34-positive microvessels of OGD showed no significant correlation with neither histological grade nor survival.

- Conclusions: The obtained results suggest that ki67, as shown here by the number of immunomarked nuclei, is an important diagnostic indicator, and can be used as a poor prognostic parameter of OGD. On the contrary, angiogenesis, considered here by the CD-34-positive microvessels, is not a prognostic indicator of ODG.

Key words: Oligodendroglioma. Angiogenesis. Cell proliferation. Ki67 antigen. CD34 antigen.

\section{Introducción}

Los oligodendrogliomas constituyen entre el 2 y el $4 \%$ de los tumores cerebrales primarios, ${ }^{1,2}$ y del 4 al $18 \%$ de los gliomas intracraneales según las diferentes series ${ }^{3,4}$, siendo más frecuentes en la edad adulta (entre 26 y 46 años) y en los varones 2,5 .

Existen diversas clasificaciones histopatológicas de estos tumores, que los dividen en cuatro grados ${ }^{6,7}$, tres grados $^{8}$ o preferentemente basado en su pronóstico dos grados, 10 , lo que coincide con la OMS que los clasifica en bien diferenciados y anaplásicos ${ }^{11,12}$.

El objetivo de este estudio es encontrar y valorar por métodos cuantitativos nuevos parámetros que nos permitan establecer el diagnóstico y el pronóstico de los oligodendrogliomas, lo que constituye uno de los retos oncológicos actuales ${ }^{13}$. Por este motivo, hemos estudiado un marcador del endotelio vascular para estudiar la angiogénesis (anticuerpo CD-34) y otro de la proliferación celular (anticuerpo anti-ki67), realizando la valoración de ambos por métodos cuantitativos.

\section{Material y métodos}

Se estudiaron 25 pacientes con lesión tumoral oligo- dendroglial (14 varones y 11 mujeres). La edad media era de 37.67 15.22 años (rango entre 23-71 años) .

Se clasificaron según sus características histopatológicas en dos grupos:

A. tumores bien diferenciados (grado II): $13 \mathrm{pa}^{-}$ cientes.

B. tumores anaplásicos (grado III): 12 pacientes.

\section{Técnicas Inmunohistológicas}

El antígeno ki67 destaca entre los antígenos de proliferación celular, ya que se ha demostrado en poblaciones celulares neoplásicas, como un excelente indicador pronóstico, debido a la correlación existente entre el marcaje del antígeno ki67 de los nucléolos durante las fases G1 , S, G2 y M (ausente en la fase G0 o estado de reposo), y el grado histopatológico de las lesiones malignas, incluidos los oligodendrogliomas ${ }^{14,15}$. Nosotros hemos podido confirmar dicha correlación en lesiones de distinto grado de malignidad en diferentes lesiones tumorales ${ }^{16,17}$.

El antígeno marcador endotelial CD-34 es una glicoproteína transmembrana de cadena única con un peso molecular de $110 \mathrm{Kd}$ que se expresa en células madres hematopoyéticas $y$, fundamentalmente, en el endotelio vascular ${ }^{18-20}$. 


\section{Inmunomarcaje Ki67}

Para la identificación de las células reactivas al suero anti-Ki67 ${ }^{16}$, se empleó la técnica inmunohistoquímica de estreptavidina-biotina (Lab. Biogenex, USA), usando como anticuerpo primario el anticuerpo monoclonal PP-67 (anti-Ki67, Lab. Sigma, USA) a dilución 1:200. Las muestras se incubaron en cámara húmeda durante 24 horas a $4^{\circ}$ centígrados. El revelado se realizó con una solucción tamponada conteniendo diaminobencidina (DAB, Lab. Sigma, USA). La reacción de oxidación de la peroxidasa fue interrumpida bajo control microscópico mediante el lavado de las preparaciones con agua corriente. El contraste se efectuó con hematoxilina. Para verificar la especificidad de la reacción, el suero anti-Ki 67 fue sustituido por PBS en lugar del anticuerpo primario con una muestra control ${ }^{16,17}$.

\section{Inmunomarcaje CD34}

Para la identificación del endotelio reactivo al suero anti-CD34 también se empleó la técnica inmunohistoquímica de estreptavidina-biotina (Lab. Biogenex, USA), usando como anticuerpo primario el anticuerpo monoclonal anti-CD34 (clon QBEND/10, Lab. Novocastra, UK) a dilución 1:50. A continuación se procede con las muestras de modo idéntico al expuesto con el anticuerpo anterior. Para verificar la especificidad de la reacción, el suero anti-CD34 fue sustituido por PBS en lugar del anticuerpo primario con una muestra control 16,17 .

\section{Estudio cuantitativo}

Todas las preparaciones fueron examinadas al microscopio óptico mediante su estudio cuantitativo. En dicha cuantificación se utilizó el programa de análisis de imagen $Q$ Win colour RGB, en un ordenador Philips con cámara de vídeo de color (Leica DC 100), utilizando siempre los mismos aumentos. Se midieron 10 campos diferentes: el área de mayor marcaje lárea caliente) y otras nueve al azar y se seleccionan los perfiles de las células, los perfiles de células ki67 positivas y los perfiles de los vasos siguiendo la metodología estandarizada ${ }^{21,22}$ y desarrollada en nuestro Departamento ${ }^{16,17}$

\section{Parámetros morfométricos}

Hemos seleccionado para su estudio estadístico los siguientes parámetros que serán las variables de estudio: número máximo de perfiles celulares (celmax); número medio de perfiles celulares (celmed); número má- ximo de perfiles celulares Ki67 positivos (celKi67max); número medio de perfiles celulares Ki67 positivos (celKi67med); número máximo de perfiles vasculares (vasmax); número medio de perfiles vasculares (vasmed); proporción entre el número máximo de células Ki67 positivas y el número máximo de células (celKi67max) celmax); proporción entre el número medio de células Ki67 positivas y el número medio de células (celKi67med/celmed); proporción entre el número máximo de perfiles vasculares y el número máximo de células (vascmax/celmax); proporción entre el número medio de perfiles vasculares y el número medio de células (vascmed/celmed).

\section{Análisis estadístico}

Grado histológico: para valorar la influencia del grado histológico del tumor, los oligodendrogliomas se clasificaron como ya hemos expuesto, en dos grupos: $A$ y $B$, según el grado de diferenciación. Una vez comprobado que las variantes eran normales, se realizó análisis de la varianza de las muestras como condicionante de validez de esta prueba paramétrica. Posteriormente, se realizó el estudio comparativo de las medias de las distintas variables cuantitativas continuas con la t de Student-Fisher.

Supervivencia: para analizar la influencia de los parámetros o variables en la supervivencia, se calcularon previamente los percentiles y cuartiles antes de aplicar el método de Kaplan-Meier para conocer los puntos de corte que puedan establecer las diferencias estadísticamente significativas del impacto clínico de esos parámetros.

Análisis multivariante: finalmente, para evaluar el valor pronóstico o predictivo de cada variable con independencia de las restantes, se empleó como prueba el modelo de la regresión múltiple de Cox.

En todas las pruebas estadísticas se emplearon los programas informáticos Microsoft Office Excel 2000 Professional y SPSS $11.0^{23}$.

\section{Resultados}

\section{Grado histológico}

- La valoración de los diferentes parámetros morfométricos según el grado histológico del tumor mostraron diferencias significativas para: celKi67máx $(p=$ 0.001 ); celKi67med ( $p=0.001$ ); celki67máx/celmáx $(p=0.001)$; celKi67med/celmed $(p=0.001)$.

El resto de los parámetros no presentaron diferencias estadísticamente significativas (Fig. 1). 


\section{A. López-Muñiz y cols.}
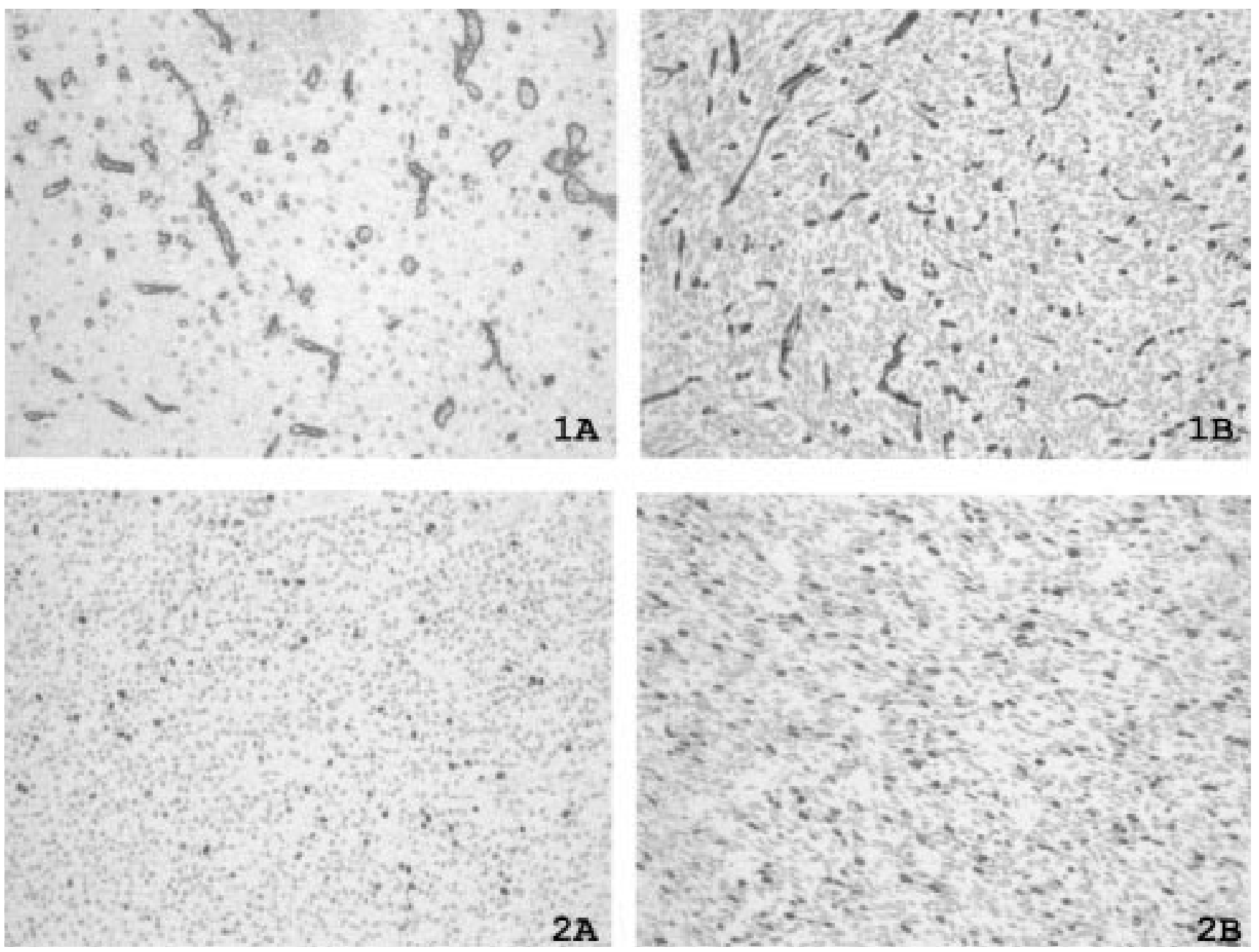

Fig. 1. Estudio Inmunocitoquímico. 1A: Observación de las estructuras vasculares en tumor bien diferenciado (marcaje antiCD34, grupo A, 40X). 1B: Demostración de la angiogénesis en tumores anaplásicos. No se descubre un aumento de las mismas (marcaje anti-CD34, grupo B, 40X). 2A: Observación de los núcleos ki67 en células de tumor bien diferenciado. (marcaje anti-ki67, grupo A, 40X). 2B: Demostración de núcleos ki67 positivos en células tumorales anaplásicas. El número y el tamaño de los núcleos están incrementados (marcaje anti-ki67, grupo B, 40X).

\section{Supervivencia}

- La influencia de las variables sobre la supervivencia demostró diferencias estadísticamente significativas para: celki67máx $(p=0.001)$; celKi67med $(p=$ 0.001 ); celKi67máx/celmáx ( $p=0.015)$; celKi67med/ celmed $(p=0.009)$.

El resto de los parámetros no presentaron diferencias estadísticamente significativas (Fig. 2).

\section{Analisis multivariante}

El estudio del valor pronóstico de cada una de las variables, con independencia de las restantes mediante la regresión de Cox, solamente mostró valor signifi- cativo para el parámetro celKi67máx/celmáx (sig.= 0.004).

Sin embargo, ninguna variable mostró diferencias significativas dentro de los oligodendrogliomas del grupo A (grado II), ni tampoco dentro de los oligodendrogliomas del grupo B (grado III).

\section{Discusión}

Hemos estudiado un marcador de la angiogénesis, debido a la importancia que el crecimiento neovascular tiene en la evolución clínica de los tumores sólidos, y un factor de proliferación celular, el ki67, por ser considerado el de mayor valor pronóstico; pero es sin 

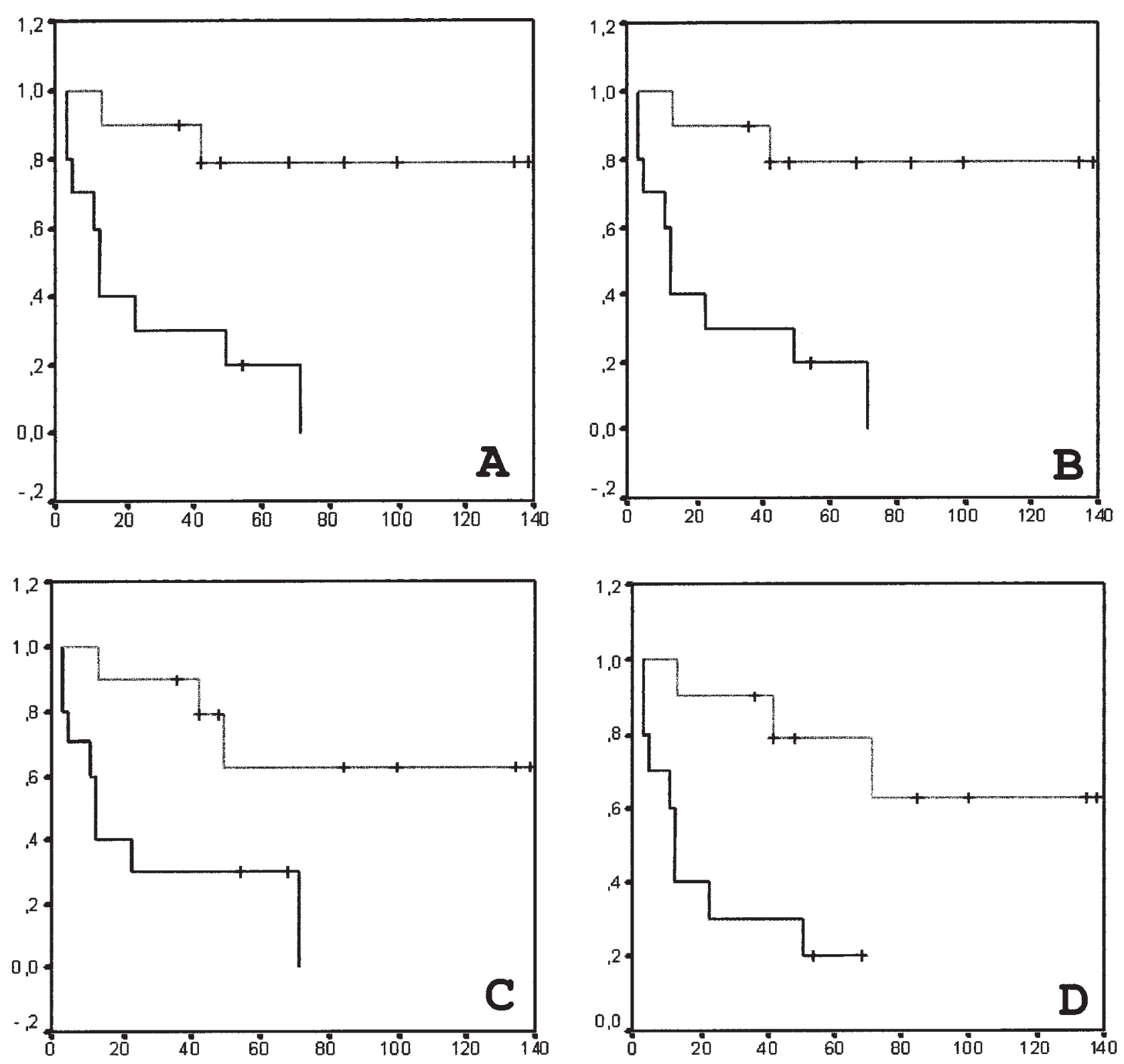

Fig. 2. Líneas de Supervivencia. A: Variable celki67max. B: Variable celki67med. C: Variable celki67max/celmax. D: Variable celki67med/celmed. (Eje $X$ supervivencia en meses. Eje $Y$ supervivencia acumulada. Línea superior subgrupo mayor de 120 meses. Línea inferior subgrupo menor de 120 meses).

duda el empleo de una técnica de valoración cuantitativa, ajena a la influencia del observador, mediante un análisis morfométrico y estereológico según la metodología desarrollada en nuestra Departamento, la principal aportación, ya que nos permite una mayor fiabilidad de los resultados.

Nuestros resultados muestran que en los pacientes con oligodendrogliomas el estudio morfométrico del material anatomopatológico de las lesiones es de enorme interés para establecer la evolución clínica del paciente, pudiendo realizarse de forma rápida y sencilla. Estos resultados demuestran que los parámetros relacionados con la angiogénesis, las mediciones vasculares, no están correlacionadas con la evolución clínica del cuadro (supervivencia) ni con el grado de malignidad. Por el contrario, la actividad proliferativa ce- 


\section{A. López-Muñiz y cols.}

lular expresada por el factor ki67, resultó un excelente marcador diagnóstico (según el grado del tumor) y pronóstico (según la supervivencia del paciente).

La microangiogénesis es considerada un requisito fundamental para el crecimiento de los tumores sólidos $^{24-26}$, estableciéndose una correlación entre algunos parámetros de la angiogénesis y la supervivencia en diversos tumores ${ }^{27-29}$ entre los que se encuentran los gliomas ${ }^{30-33}$. Sin embargo, los oligodendrogliomas parecen ser una excepción, comprobándose que pueden crecer lentamente durante años en forma de células neoplásicas que infiltran el parénquima cerebral ${ }^{30}$ y en un posterior periodo tener lugar la microangénesis en áreas del parénquima de alta densidad tumo$\mathrm{ral}^{1,30}$. Por este motivo algunos estudios, coincidiendo con nuestras observaciones, discrepan sobre la valoración del contaje de los vasos y la supervivencia de los pacientes $^{34-36}$, aunque otros sí han relacionado el número de vasos por unidad, con la supervivencia ${ }^{37-38}$.

Por el contrario, en la actualidad los autores coinciden en destacar la importancia del factor ki67 en el pronóstico de los oligodendrogliomas, por tratarse del más sensible de los indicadores de la proliferación celular ${ }^{39-45}$. Nuestro trabajo ratifica estos hallazgos tras un exhaustivo análisis morfométrico y estereológico.

En consecuencia, nuestro trabajo confirma que el factor de proliferación celular Ki67 debería realizarse en los oligodendrogliomas por su importancia en la valoración de la supervivencia de los pacientes, sugerencia compartida en recientes trabajos ${ }^{46-48}$. Por el contrario, en este tipo de tumores el estudio de la angiogénesis y de los marcadores endoteliales tendría escaso interés para establecer un pronóstico, pudiendo ser descartados. Consecuentemente, sugerimos que un riguroso análisis morfométrico debe ser añadido a la rutina clínica en la valoración de los oligodendrogliomas.

\section{Bibliografía}

1. Daumas-Duport C, Tucker ML, Kolles H, et al. Oligodendrogliomas. A new grading system based on morphological and imaging criteria. J Neurooncol. 1997; 34(1): 61-78.

2. Mork SJ, Lindegaard KF, Halvorsen TB, et al. Oligodendroglioma: incidence and biological behavior in a defined population. J Neurosurg. 1985; 63(6):881-9.

3. Schiffer D. Brain Tumors. Biology, Pathology and Clinical references. Berlin, Ed.: Springer-Verlag. 2a Edition. 1997.

4. Zulch KJ. Brain Tumors. Their Biology and Pathology. Berlin. Ed.: Springer-Verlag. $3^{a}$ Edition. 1986.

5. Chin HW, Hazel JJ, Kim TH, Webster JH. Oligodendrogliomas. I. A clinical study of cerebral oligodendrogliomas. Cancer. 1980; 45(6):1458-66.

6. Shaw EG, Scheithaver BW, O'Fallon JR, Tazelaar HD, Davis $\mathrm{DH}$. Oligodendrogliomas: the Mayo Clinic experience. J Neurosurg. 1992; 76(3):428-34.

7. Smith $M T$, Ludwig CL, Godfrey AD, Armbrustmacher WW. Grading of oligodendrogliomas. Cancer. 1983; 52 (11):2107-14.

8. Kros JM, Pieterman H, van Eden CG, Avezaat CJ. Oligodendroglioma: the Rotterdam-Dijkzigt experience. Neurosurgery. 1994; 34(6):959-66;

9. Kros JM, Troost D, van Eden CG, van $\operatorname{der}$ Werf AJ, Uylings $\mathrm{HB}$. Oligodendroglioma. A comparison of two grading systems. Cancer. 1988; 61(11):2251-9.

10. Nijiar TS, Simpson WJ, Gadalla T, McCartney M. Oligodendroglioma. The Princess Margaret Hospital experience (1958-1984). Cancer. 1993; 71(12):4002-6.

11. Dehghani F, Schachenmayr W, Laun A, Korf HW. Prognostic implication of histopathological, immunohistochemical and clinical features of oligodendrogliomas: a study of 89 cases. Acta Neuropathol. 1998; 95(5):493504.

12. Wharton SB, Chan KK, Hamilton FA, Anderson JR. Expression of neuronal markers in oligodendrogliomas: an immunohistochemical study. Neuropathol Appl Neurobiol. 1998; 24(4):302-8.

13. Hagel C, Krog B, Laas R, Stavrou DK. Prognostic relevance of TP53 mutations, p53 protein, Ki67 index and conventional histological grading in oligodendrogliomas. J Exp Clin Cancer Res. 1999; 18(3):305-9.

14. Brown DC, Gatter KC. Monoclonal antibody ki67: its use in histopathology. Histopathology. 1990; 17:489-503.

15. Coons SW, Johnson PC, Pearl DK. The prognostic significance of Ki67 labeling indices for oligodendrogliomas. Neurosurgery. 1997 Oct;41(4):878-84/5.

16. García -Pola MJ, Anitua MJ, Fernández BE, Garcia M, López-Muñiz A. Study comparative of ki67 expression in oral lichen planus and oral leukoplakia. Quantitative analysis. Med Oral. 2001; 6:384-70.

17. López-Muñiz A, Triviño A, García Fernández C, Gil JA, 
Herrero A. Análisis cuantitativo e la expresión ki67 en lesiones melanocíticas benignas y malignas. Oncología. 2003; 26(2):79-84.

18. Parums DV, Cordell JL, Micklem K, Heryet AR, Gatter KC, Mason DY. JC70: a new monoclonal antibody that detects vascular endothelium associated antigen on routinely processed tissue sections. J Clin Pathol. 1990; 43(9):752-7.

19. Li VW, Folkerth RD, Watanabe $\mathrm{H}$, et al. Microvessel count and cerebrospinal fluid basic fibroblast growth factor in children with brain tumours. Lancet. 1994; 344(8915):82-6.

20. Ramani P, Bradley NJ, Fletcher CD. QBEND/10, a new monoclonal antibody to endothelium: assessment of its diagnostic utility in paraffin sections. Histopathology. 1990; 17(3):237-42.

21. Gundersen HJ, Bagger P, Bendtsen TF, et al. The new stereological tools: disector, fractionator, nucleator and point sampled intercepts and their use in pathological research and diagnosis. APMIS. 1988; 96(10):85781.

22. Gundersen HJ, Bendtsen TF, Korbo L, et al. Some new, simple and efficient stereological methods and their use in pathological research and diagnosis. APMIS. 1988; 96(5):379-94

23. Kleinbaum DG, Kuipper LL, Muller KE. Basic statistics, the correlation coefficient and straighline analysis. En: Kent PWA, ed. Applied regression analysis and other multivariate methods. Boston: PW Kent, 1998; 335-717.

24. Folkman J. Tumor angiogenesis: therapeutic implications. N Engl J Med. 1971; 285(21):1 182-6.

25. Folkman J, Klagsbrun M. Angiogenic factors. Science. 1987; 235(4787):442-7.

26. Folkman J. What is the evidence that tumors are angiogenesis dependent? J Natl Cancer Inst. 1990; 82(1):4-6.

27. Srivastava A, Laidler P, Davies RP, Horgan K, Hughes $L E$. The prognostic significance of tumor vascularity in intermediate-thickness (0.76-4.0 mm thick) skin melanoma. A quantitative histologic study. Am J Pathol. 1988; 133(2):419-23.

28. Porschen R, Classen S, Piontek M, Borchard F. Vascularization of carcinomas of the esophagus and its correlation with tumor proliferation. Cancer Res. 1994; 54(2): 587-91.

29. Weidner N, Semple JP, Welch WR, Folkman J. Tumor angiogenesis and metastasis-correlation in invasive breast carcinoma. N Engl J Med. 1991; 324(1):1-8.

30. Daumas-Duport C, Varlet $P$, Tucker ML, Beuvon F, Cervera $\mathrm{P}$, Chodkiewicz JP. Oligodendrogliomas. Part I: Patterns of growth, histological diagnosis, clinical and imaging correlations: a study of 153 cases. J Neurooncol. 1997; 34(1):37-59.

31. Berkman RA, Merrill MJ, Reinhold WC et al. Expression of the vascular permeability factor/vascular endothelial growth factor gene in central nervous system neoplasms. J Clin Invest. 1993; 91(1):153-9.
32. Plate $\mathrm{KH}$, Risau W. Angiogenesis in malignant gliomas. Glia. 1995; 15(3):339-47.

33. Abdulrauf SI, Edvardsen $\mathrm{K}, \mathrm{Ho} \mathrm{KL}$, Yang XY, Rock JP, Rosenblum ML. Vascular endothelial growth factor expression and vascular density as prognostic markers of survival in patients with low-grade astrocytoma. J Neurosurg. 1998; 88(3):513-20.

34. Vaquero J, Zurita M, Coca S, Oya S, Morales C. Prognostic significance of clinical and angiogenesis-related factors in low-grade oligodendrogliomas. Surg Neurol. 2000; 54(3):229-34.

35. Vaquero J, Zurita M, Morales C, Coca S. Prognostic significance of the endothelial surface in low-grade resected oligodendrogliomas. Br J Neurosurg. 2001; 15(3): 247-50.

36. Vaquero J, Zurita M, Morales C, Coca S. Prognostic significance of tumor-enhancement and angiogenesis in oligodendroglioma. Acta Neurol Scand. 2002; 106(1):1923.

37. Leon SP, Folkerth RD, Black PM. Microvessel density is a prognostic indicator for patients with astroglial brain tumors. Cancer. 1996; 77(2):362-72.

38. Korkolopoulou P, Patsouris E, Kavantzas, et al. Prognostic implications of microvessel morphometry in diffuse astrocytic neoplasms. Neuropathol Appl Neurobiol. 2002; 28(1):57-66.

39. Fortin D, Cairncross GJ, Hammond RR. Oligodendroglioma: an appraisal of recent data pertaining to diagnosis and treatment. Neurosurgery. 1999; 45(6):127991.

40. Kros JM, Hop WC, Godschalk JJ, Krishnadath KK. Prognostic value of the proliferation-related antigen Ki67 in oligodendrogliomas. Cancer 1996; 78(5):1 107-13.

41. Heegaard S, Sommer HM, Broholm H, Broendstrup O. Proliferating cell nuclear antigen and Ki67 immunohistochemistry of oligodendrogliomas with special reference to prognosis. Cancer. 1995; 76(10):1809-13.

42. Rodriguez-Pereira C, Suarez-Penaranda JM, VazquezSalvado M, et al. Value of MIB-1 labelling index (LI) in gliomas and its correlation with other prognostic factors. A clinicopathologic study. J Neurosurg Sci. 2000; 44(4):203-9.

43. Wharton SB, Chan KK, Anderson JR, Stoeber K, Williams GH. Replicative Mcm2 protein as a novel proliferation marker in oligodendrogliomas and its relationship to Ki67 labelling index, histological grade and prognosis. Neuropathol Appl Neurobiol. 2001; 27(4):305-13.

44. Marie D, Liu Y, Moore SA, et al. Interobserver variability associated with the MIB-1 labeling index: high levels suggest limited prognostic usefulness for patients with primary brain tumors. Cancer. 2001; 92(10):2720-6.

45. Prayson RA, Castilla EA, Hembury TA, Liu W, Noga $\mathrm{CM}$, Prok AL. Interobserver variability in determining MIB-1 labeling indices in oligodendrogliomas. Ann Diagn Pathol. 2003; 7(1):9-13. 


\section{A. López-Muñiz y cols.}

46. Reis-Filho JS, Faoro LN, Carrilho C, Bleggi-Torres LF, Schmitt FC. Evaluation of cell proliferation, epidermal growth factor receptor, and bcl-2 immunoexpression as prognostic factors for patients with World Health Organization grade 2 oligodendroglioma. Cancer. 2000; 88(4):862-9.

47. Giannini C, Scheithaver BW, Weaver AL, et al. Oligo- dendrogliomas: reproducibility and prognostic value of histologic diagnosis and grading. J Neuropathol Exp Neurol. 2001; 60(3):248-62.

48. Kayaselcuk F, Zorludemir S, Gumurduhu D, Zeren H, Erman T. PCNA and Ki67 in central nervous system tumors: correlation with the histological type and grade. J Neurooncol. 2002; 57(2):115-21. 